\title{
Probing the Magnetic Field Structure in Gamma\# Ray Bursts through Dispersive Plasma Effects on the Afterglow Polarization
}

\section{Citation}

Sagiv, Amir, Eli Waxman, and Abraham Loeb. 2004. "Probing the Magnetic Field Structure in Gamma\#Ray Bursts through Dispersive Plasma Effects on the Afterglow Polarization." The Astrophysical Journal 615 (1): 366-77. https://doi.org/10.1086/423977.

\section{Permanent link}

http://nrs.harvard.edu/urn-3:HUL.InstRepos:41393385

\section{Terms of Use}

This article was downloaded from Harvard University's DASH repository, and is made available under the terms and conditions applicable to Other Posted Material, as set forth at http:// nrs.harvard.edu/urn-3:HUL.InstRepos:dash.current.terms-of-use\#LAA

\section{Share Your Story}

The Harvard community has made this article openly available. Please share how this access benefits you. Submit a story.

Accessibility 
The Astrophysical Journal, 615:366-377, 2004 November 1

(C) 2004. The American Astronomical Society. All rights reserved. Printed in U.S.A.

\title{
PROBING THE MAGNETIC FIELD STRUCTURE IN GAMMA-RAY BURSTS THROUGH DISPERSIVE PLASMA EFFECTS ON THE AFTERGLOW POLARIZATION
}

\author{
Amir Sagiv, ${ }^{1}$ Eli Waxman, ${ }^{1}$ And Abraham Loeb ${ }^{2,3}$ \\ Received 2004 January 29; accepted 2004 July 6
}

\begin{abstract}
The origin and structure of magnetic fields in gamma-ray burst (GRB) fireball plasmas are two of the most important open questions in all GRB models. It has been claimed that recent measurements of $\gamma$-ray polarization suggest the presence of a uniform field originating in the compact object driving the outflow. This interpretation is, however, controversial, since a high degree of linear polarization is also possible in the presence of a random magnetic field, arguably originating in electromagnetic instabilities that develop at the collisionless shock. We show that the structure and strength of the magnetic field may be constrained by radio and IR observations of the early afterglow, where plasma effects on the polarization of the propagating radiation are significant. We calculate these propagation effects for cold and relativistic plasmas, and find that in the presence of a uniform equipartition field the degree of linear polarization is suppressed and circular polarization prevails at low frequencies, below $\sim 1-3 \mathrm{GHz}\left(\sim \mathrm{a}\right.$ few $\left.\times 10^{14} \mathrm{~Hz}\right)$ in the forward (reverse) shock, at the onset of fireball deceleration. At higher frequencies linear polarization dominates. At the frequency of the transition between circular and linear polarization, the net level of polarization is minimal, $\sim 10 \%-20 \%$. These features are nearly independent of the density of the environment into which the fireball expands. When the uniform field is much weaker than the equipartition value, the transition frequency is smaller by an order of magnitude. Depending on the geometry of the emitting region, oscillations of the polarization position angle may be observed from the optical reverse-shock emission, provided that the strength of the magnetic field is close to equipartition. The dependence of these results on viewing geometry, outflow collimation, and magnetic field orientation is discussed. When the field is entangled over length scales much smaller than the extent of the emitting plasma, the aforementioned effects should not be observed, and a linear polarization at the few percent level is expected. Polarimetric observations during the early afterglow, and particularly of the reverse-shock emission, may therefore place strong constraints on the structure and strength of the magnetic field within the fireball plasma.
\end{abstract}

Subject headings: gamma rays: bursts — magnetic fields — plasmas — polarization — shock waves

\section{INTRODUCTION}

The past decade has been marked by major advances in our understanding of gamma-ray bursts (GRBs). These bright explosions at cosmological distances are now commonly believed to be generated by the dissipation of a relativistic outflow associated with the gravitational collapse of a stellar mass object to a compact remnant (for reviews, see Piran 2000; Mészáros 2002; Waxman 2003a). Observations suggest that the outflow is probably collimated to within a narrow jet, with smaller opening angles corresponding to apparently more energetic bursts (Frail et al. 2001). Current models relate the production of $\gamma$-rays to emission of synchrotron or inverseCompton radiation by relativistic electrons. Subsequently, the interaction of the ejecta with the circumburst gas drives a forward shock into the ambient medium, and a reverse shock into the ejecta. This stage during which the forward and reverse shocks coexist is usually dubbed the "transition phase"; once the reverse shock fully crosses the ejecta, the dynamics of the expanding fireball is adequately described by a self-similar solution (Blandford \& McKee 1976). During the self-similar phase that follows, a forward shock propagates in the ambient gas, gradually decelerating, until the inverse of the shock

\footnotetext{
${ }^{1}$ Physics Faculty, Weizmann Institute of Science, Rehovot, Israel; amir@ wicc.weizmann.ac.il.

2 Astronomy Department, Harvard University, 60 Garden Street, Cambridge, MA 02138 .

3 Einstein Minerva Center, Physics Faculty, Weizmann Institute of Science, Rehovot, Israel.
}

Lorentz factor drops below the jet opening angle (indicating the "jet break time"), at which stage the jet quickly slows down and spreads sideways. Subsequently the expansion continues subrelativistically. Electrons accelerated at these shocks emit synchrotron radiation, which appears as an optical flash from the reverse shock (Mészáros et al. 1994; Akerlof et al. 1999; Zhang et al. 2003, and reference therein), lasting typically for the duration of the GRB itself, as well as a long-lived transient afterglow from the forward shock, characterized by a decreasing peak frequency, which lasts for weeks or even months (Frail et al. 2000).

Despite the successes of the models in predicting and explaining light curves and fluxes at various epochs and various wavebands, key questions, concerning predominantly the nature of the progenitor, the launching of the relativistic outflow, and the physics of the relativistic shock waves, are still unanswered. In addition, the physical mechanisms responsible for the production of $\gamma$-rays are still subject to controversy. Leading scenarios for the production of the prompt $\gamma$-ray emission can be grouped into three main categories. The first consists of models in which the relativistic outflow is dominated by the kinetic energy of the ejecta, and the $\gamma$-rays are the consequence of synchrotron emission by relativistic electrons, which are Fermi-accelerated in optically thin collisionless shocks created by inhomogeneities in the ejecta ("internal shocks"). The assumption that the $\gamma$-rays are synchrotron photons requires strong magnetic fields, which would probably result from the amplification of a small-scale field by plasma instabilities in collisionless shocks (Gruzinov \& Waxman 1999; Medvedev 
\& Loeb 1999). In this scenario, the coherence scale of the magnetic field may grow to the size of the causally connected regions in the outflow, a size that is still smaller than the scale of the emitting slab. Afterglow observations strongly favor the formation of strong magnetic fields at relativistic shock fronts, and may therefore indicate that such a mechanism is also governing the emission from the internal shocks. In a second type of model the outflow is Poynting-flux dominated, and current-driven instabilities at large radii lead to acceleration of pairs that emit $\gamma$-rays by synchrotron radiation (Lyutikov et al. 2003). The third type of model associates the prompt emission with the relativistic boosting of an ambient photon field, possibly related to an earlier supernova explosion (Lazzati et al. 2000), by electrons outflowing with a high Lorentz factor.

The recent detection of a strong linear polarization signal in the prompt $\gamma$-ray emission of GRB 021206 by Coburn \& Boggs (2003) triggered much interest in GRB polarization features (but see Rutledge \& Fox [2004], who critically reanalyze the light curve of GRB 021206, and find no polarization signal). This measurement - if real-provided a strong indication that synchrotron emission is indeed the underlying radiation mechanism of the GRB phenomenon (Waxman 2003b). Its interpretation in terms of magnetic field structure is, however, ambiguous. It has been claimed that the high polarization in $\gamma$-rays suggests the presence of a uniform magnetic field in the emitting plasma (Lyutikov et al. 2003), thus supporting the paradigm of a Poynting-flux dominated outflow advecting a "primordial" field from the "inner engine." It turns out, however, that this line of reasoning is not free of caveats. First, it was recently demonstrated (Waxman 2003b; Granot 2003; Nakar et al. 2003) that an outflow that is collimated to within a narrow jet and is observed off-axis may exhibit a high degree of linear polarization, even in the presence of a random magnetic field. Second, even the presence of a uniform field is still not an indication that the outflow is Poynting-flux dominated, since the amplitude of a toroidal magnetic field decreases as $R^{-1}$ and tends to dominate at large radii-even if the outflow is dominated by kinetic energy. The uniform field hypothesis is controversial also since it appears difficult to reconcile the existence of a large-scale magnetic field with the presence of relativistic charge particles, which are accelerated in situ, since current understanding of particle acceleration requires the dissipation of these large-scale fields.

In this paper we examine the fireball scenario, in which the outflow is dominated by the plasma kinetic energy, and demonstrate that the strength and structure of the magnetic field may be constrained by radio and IR observations of the early afterglow, where plasma effects on the propagation of synchrotron radiation are significant. Specifically, we show that the presence of a uniform field permeating the plasma has distinctive fingerprints on the polarization properties of emitted radiation, which distinguish it from the case of a field entangled over small length scales. Of particular interest is the effect on the polarization of the reverse-shock emission, since that plasma is just the ejecta producing the prompt $\gamma$-ray emission. Hence, constraining the structure and strength of the magnetic field in the reverse shock is complementary to measurements of the $\gamma$-ray polarization, and has direct implications for the plasma conditions in the ejecta and consequently for processes taking place in the vicinity of the compact source. This powerful probe may help to put constraints on models of field generation, thus promoting our understanding of both the shock physics and the nature of the "inner engine."
This article is organized as follows. In $\oint 2$ we provide a brief review of polarized light transfer in a magnetoactive plasma, along with new results for a relativistic plasma. Next, in $\S 3$, follows a qualitative discussion of fireball geometry and observation geometry, in the context of plasma propagation effects. The propagation effects are applied to GRB plasmas in $\S 4$. In $\S 4.1$ we obtain plasma conditions during the early afterglow, considering cases of both an expansion into a uniform-density interstellar medium (ISM), in $\S 4.1 .1$, and an expansion into a circumburst wind, in $\S 4.1 .2$. Detailed analysis of propagation effects at the corresponding shocks follows in $\S$ 4.2. The implications of the strength and structure of the magnetic field are discussed in $\S 4.4$. Finally, we consider the implications of our results in $\S 5$.

\section{PLASMA EFFECTS ON THE POLARIZATION OF PROPAGATING SYNCHROTRON RADIATION}

As is well known, a plasma permeated by a magnetic field is birefringent, and a phase shift accumulates between the normal modes as radiation propagates through the plasma. The observational outcome of this phenomenon is the Faraday rotation effect. Below we briefly review the formalism of transfer of polarized synchrotron radiation in a magnetoactive plasma ( $\S 2.1$ ), and bring new results for a plasma dominated by a relativistic electron population ( $\S 2.2$ ). The Appendix gives a more comprehensive derivation of these results.

\subsection{Formalism}

The effect of the magnetoactive plasma on the transfer of polarized radiation can be treated most straightforwardly via the analysis of the wave equation, incorporating the effect of the medium by a dielectric tensor. When the radiation can be regarded as transverse, the tensor $\kappa_{i j}(i, j=1,2),{ }^{4}$ representing the plasma effect in the dielectric tensor, is separated into a sum of Hermitian and anti-Hermitian tensors as

$$
4 \pi \frac{\omega}{c} \kappa_{i j}=\left(\begin{array}{cc}
h & \text { if } \\
-i f & -h
\end{array}\right)+i\left(\begin{array}{cc}
\kappa+q & i v \\
-i v & \kappa-q
\end{array}\right) .
$$

The anti-Hermitian component of the tensor is responsible for absorption. The Hermitian part is responsible for propagation effects; specifically, $f=\Delta k(\omega)=\omega \Delta n(\omega) / c$ is the difference between the wavenumbers of the normal modes, giving rise to the Faraday rotation effect. In the case of synchrotron selfabsorption, the parameter $v$ is typically smaller than $\kappa$ by a factor $\gamma_{m}$, where $\gamma_{m}$ is a typical Lorentz factor of the relativistic electrons, and will therefore be neglected hereafter.

The wave equation for the Fourier components of the electric field can be manipulated, with the help of equation (1), into a transfer equation for the Stokes parameters:

$$
\begin{aligned}
d I / d s & =\varepsilon_{I}-\kappa I-q Q \\
d Q / d s & =\varepsilon_{Q}-q I-\kappa Q-f U \\
d U / d s & =f Q-\kappa U-h V \\
d V / d s & =\varepsilon_{V}+h U-\kappa V,
\end{aligned}
$$

where $s$ is a length parameter along the path of the ray, and $\varepsilon_{I}$, $\varepsilon_{Q}$, and $\varepsilon_{V}$ are the emission coefficients corresponding to $I, Q$, and $V$, respectively.

\footnotetext{
${ }^{4}$ We adopt a right-handed system of coordinates with the wavevector $\boldsymbol{k}$ directed along axis 3 .
} 
The physical significance of the propagation parameters $f$ and $h$ is most easily demonstrated in a transparent medium. As an example, consider the case $f \gg h$, with negligible $\kappa$ and $q$. Equation (1) then implies that the normal modes are (left- and right-) circularly polarized. Integrating equation (2), we obtain oscillations in the Stokes parameters $Q$ and $U$, leading to oscillations and damping in the degree of emergent linear polarization,

$$
\Pi_{L}=\frac{\sqrt{Q^{2}+U^{2}}}{I} \simeq \frac{\epsilon_{Q}}{\epsilon_{I}}\left|\frac{\sin \left(f_{S} / 2\right)}{f_{S} / 2}\right| .
$$

A large phase shift accumulated between the normal modes, $\Delta \phi=f_{S} \gg 1$, results in damping of the degree of linear polarization by a factor of $\Delta \phi$. This is accompanied by oscillations of the polarization position angle $\chi=\frac{1}{2} \tan ^{-1}(U / Q)$, providing the Faraday rotation effect. Analogous, simple-tointerpret phenomena also characterize the case $h \gg f$ (linearly polarized normal modes).

\subsection{New Results for Relativistic Plasma}

The coefficients $f$ and $h$ are easily derived when the plasma is cold:

$$
\begin{aligned}
f_{\text {cold }} & =\frac{\tilde{\omega}_{p}^{2} \tilde{\omega}_{B} \cos \vartheta}{c\left(\omega^{2}-\tilde{\omega}_{B}^{2}\right)} \\
h_{\text {cold }} & =\frac{\tilde{\omega}_{p}^{2} \tilde{\omega}_{B}^{2} \sin ^{2} \vartheta}{2 c \omega\left(\omega^{2}-\tilde{\omega}_{B}^{2}\right)},
\end{aligned}
$$

where $\tilde{\omega}_{p}^{2}=4 \pi n_{e} e^{2} / m_{e}$ is the nonrelativistic electron plasma frequency, $\tilde{\omega}_{B}=e B / m_{e} c$ is the nonrelativistic electron Larmor frequency, and $\vartheta$ is the angle between $\boldsymbol{k}$ and $\boldsymbol{B}$, measured in the frame comoving with the plasma.

In the context of GRB plasmas, however, one also encounters a situation in which the plasma is dominated by a relativistic electron population. In our subsequent derivation of analytic expressions for the propagation coefficients in a relativistic plasma we assume that (1) the electrons are highly relativistic, characterized by a spectrum with a power-law tail extending to high energies; (2) their distribution function is isotropic; and (3) the deviation of the dielectric tensor from its vacuum value is small. The first assumption is implied by afterglow observations, whereas the second is adopted for simplicity. The third assumption will be justified later for the specific parameters of GRB plasmas. In addition, we restrict the discussion to a frequency range $\omega \gg \omega_{B}$, so that modifications to synchrotron emission and self-absorption coefficients due to the discrete nature of low harmonics of $\nu_{B}$ could be safely ignored.

Our main results are summarized in the following equations (for an outline of the derivation, see Appendix A.1):

$$
\begin{aligned}
& f_{\mathrm{rel}}(\nu) \simeq \frac{\tilde{\omega}_{p}^{2} \tilde{\omega}_{B} \cos \vartheta}{c \omega^{2}} \frac{\ln \gamma_{m}}{\gamma_{m}^{2}}=\frac{e^{3} n_{e} B \cos \vartheta}{\pi m_{e}^{2} c^{2} \nu^{2}} \frac{\ln \gamma_{m}}{\gamma_{m}^{2}}, \\
h_{\mathrm{rel}} \simeq & \frac{e^{4} n_{e} B^{2} \sin ^{2} \vartheta}{4 \pi^{2} m_{e}^{3} c^{3} \nu^{3}} \\
& \times \begin{cases}\frac{\gamma_{m}}{2}\left(\frac{\nu}{\nu_{m}}\right)^{4 / 3} & \text { if } \nu \ll \nu_{m}, \\
\left(\frac{2}{p-2}\right) \gamma_{m}^{-(p-2)}\left(\frac{\nu}{\nu_{m}}\right)^{(p-2) / 2} & \text { if } \nu>\nu_{m} .\end{cases}
\end{aligned}
$$

Here $\nu=\omega / 2 \pi, \gamma_{m}$ is a characteristic electron Lorentz factor (typically that of the thermal electrons), $\nu_{m}=(3 e B) /\left(4 \pi m_{e} c\right) \gamma_{m}^{2}$ is the characteristic synchrotron frequency corresponding to $\gamma_{m}$, and $p$ is the spectral index of the power-law tail of the electron distribution function. Equation (6) generalizes an earlier result by Sazonov (1969), who considered only the frequency range $\nu \gg \nu_{m}$.

After the completion of this work, we learned of an independent derivation of similar expressions for the propagation coefficients in the relativistic regime by Matsumiya \& Ioka (2003). Our expressions (eqs. [5] and [6]) agree nicely with theirs; nevertheless, the resulting polarization differs substantially, as we further discuss in $\S 5$.

Using these results in the context of Faraday rotation, we notice that $f$, and therefore also $\Delta \phi$, are proportional to $\nu^{-2}$. The frequency interval $\Delta \nu$, corresponding to a single period of the position angle oscillation, translates to an increase of $2 \pi$ in $\Delta \phi$, from which we get (for a cold plasma)

$$
\left|\frac{\Delta \nu}{\nu}\right|=\frac{\pi^{2} m_{e}^{2} c^{2} \nu^{2}}{e^{3} n_{e} B W},
$$

where $W$ is the width of the slab through which the radiation propagates. The generalization for a relativistic plasma is trivial.

\section{CONSEQUENCES OF FIREBALL GEOMETRY AND VIEWING GEOMETRY}

A strong polarization signal clearly requires a departure from spherical symmetry, either via a nonspherical outflow configuration and a special location of the observer with respect to it, or through a preferred direction of the magnetic field in the plasma (or, of course, a combination of the two). Geometrical considerations related to the fireball collimation, viewing angle, and field direction are essential for the calculations we describe below, and so before presenting detailed results of these calculations we make a short digression and discuss qualitatively some important consequences of the geometry. In particular, we show that the angle $\vartheta$, on which the propagation coefficients $f$ and $h$ depend (cf. eqs. [4]-[6]) is not likely to assume special values such as 0 and $\pi / 2$ in a typical GRB jet, and may approach 0 only for a narrow jet observed off-axis, with a magnetic field oriented normal to the shock plane; these two regimes have markedly different observational consequences. We focus in this section only on the case of a large-scale, uniform magnetic field. The case of a fluctuating field is treated in $\S 4.4$.

Figure 1 shows schematically the essential ingredients of the fireball and viewing geometry. The figure also illustrates (shaded areas) electron cooling due to synchrotron losses away from the (forward and reverse) shock fronts. This effect, which has important consequences for the resulting polarization, is discussed in greater detail in $\S 4.2$.

Mounting evidence strongly suggests that the relativistic outflow in GRBs is collimated into a jet, the opening angle of which is usually inferred based on estimates of the jet break time from optical observations (Frail et al. 2001). These observations are carried on a day timescale after the GRB trigger, when the jet has already decelerated to a low Lorentz factor, $\Gamma \sim 10$, whence one deduces $\theta_{j} \sim 0.1$. We, on the other hand, are interested in the emission from the early afterglow, at which stage the fireball expands with a much higher Lorentz factor, $\Gamma \sim 10^{2.5}$ (see eqs. [8] and [14]). It is unclear whether during this early stage the jet opening angle is similar to the one inferred from optical observations. However, Freedman \& 


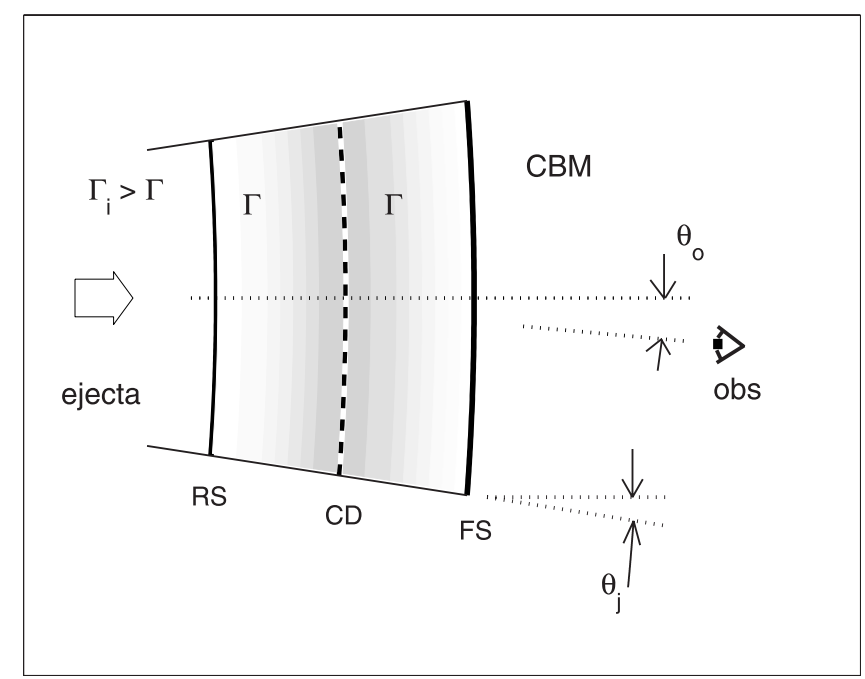

FIG. 1.-Schematic illustration of the fireball and viewing geometry; $\theta_{j}$ and $\theta_{o}$ are, respectively, the jet opening angle and the angle between the line of sight to the observer and the jet axis; $\Gamma_{i}$ is the Lorentz factor of the ejecta prior to deceleration, and $\Gamma$ is the Lorentz factor of the plasma shocked by the reverse and forward shocks. The initials RS, CD, and FS denote the reverse shock, the contact discontinuity, and the forward shock, respectively, while CBM denotes the circumburst material. The dark shading marks the cooling of the electrons, with darker shade representing colder electrons.

Waxman (2001) have demonstrated that the isotropic-equivalent fireball energy can be robustly estimated from the X-ray afterglow flux on a day timescale after the GRB, and furthermore that this estimate exhibits a strong correlation with the isotropicequivalent energy inferred from the GRB $\gamma$-ray fluence (see also Berger et al. [2003] for a recent analysis compiling more X-ray data). This correlation, and in particular the fact that the latter estimate is never much larger than the former, strongly argue in favor of a similar jet opening angle during the GRB and early afterglow phases. Since the X-ray afterglow is obtained on a day timescale, when the jet has already decelerated to $\Gamma \sim 10$, this arguments suggests an opening angle $\theta_{j} \sim 0.1$ for a typical GRB jet. An observer of the early afterglow is therefore not likely to see the edge of a typical jet, and is consequently not sensitive to deviations of the fireball from spherical symmetry (other than a large-scale magnetic field in a particular direction).

Deviations from spherical symmetry may be manifested for an observer located on a line of sight that makes an angle $\sim \Gamma^{-1}$ from the jet edge, and are therefore probable only if the jet is extremely narrow, with $\theta_{j} \sim \Gamma^{-1}$. During the GRB and early afterglow phases the fireball expands with $\Gamma \sim 10^{2.5}$, and so the arguments presented above suggest that such narrow jets are not generic. However, in the framework of the "standard energy reservoir" hypothesis promoted by Frail et al. (2001), brighter GRBs are naturally associated with narrower jets. Adopting this energy-angle relation, GRB 021206, which was among the strongest bursts ever, had an opening angle $<1 / 40 \mathrm{rad}$, or even smaller (Nakar et al. 2003). This observation is key in associating the high degree of linear polarization measured in GRB 021206 with an observational alignment effect (Waxman 2003b; Nakar et al. 2003).

Upon transforming to the frame comoving with the fireball, the distinct geometries of a "typical jet" and a "narrow jet" correspond to the radiation $\boldsymbol{k}$ vector making either a large $\left(\gg \Gamma^{-1}\right)$ or a small $\left(\sim \Gamma^{-1}\right)$ angle relative to the plane of the shock, for typical $\left(\theta_{j} \gg \Gamma^{-1}\right)$ and narrow $\left(\theta_{j} \sim \Gamma^{-1}\right)$ jets, respectively. Assume now that the magnetic field is dominated by a component either parallel or normal to the plane of the shock. The angle $\vartheta$ between $\boldsymbol{k}$ and $\boldsymbol{B}$ may assume special values, i.e., close to 0 or to $\pi / 2$ (see an illustration of these two geometries in Fig. 2). Recalling the angular dependencies of the propagation coefficients, $f \propto \cos \vartheta$ and $h \propto \sin ^{2} \vartheta$ (approximately; see eq. [6]), this may result in very different propagation
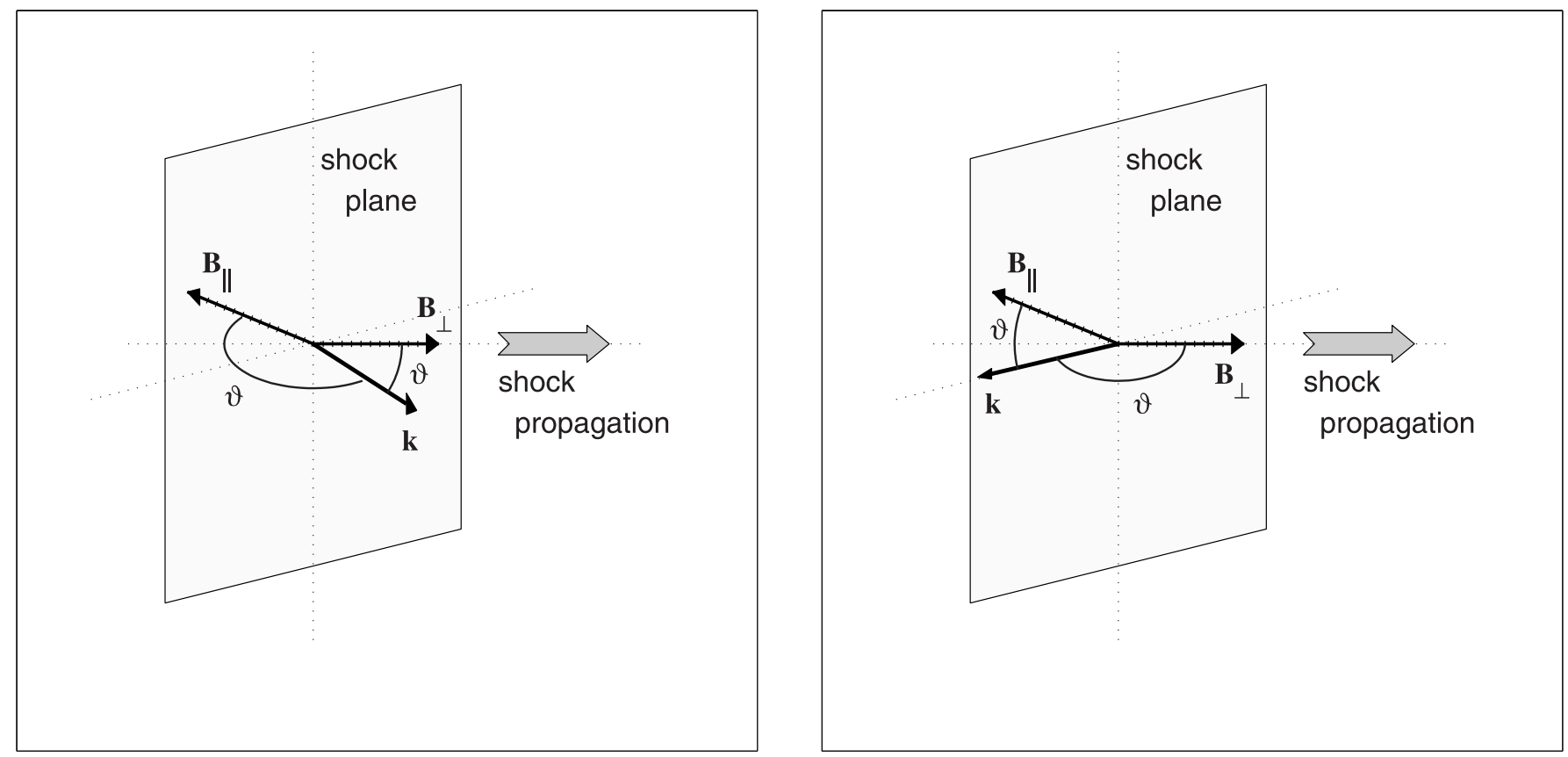

FIG. 2.-Geometrical setup in the frame comoving with the plasma. Shown are $\boldsymbol{k}$, the direction of the radiation wavevector (i.e., the line of sight); $\boldsymbol{B}_{\perp}$, a component of the magnetic field normal to the shock plane; and $\boldsymbol{B}_{\|}$, the field component lying in the shock plane. The angle $\vartheta$ between the line of sight and the field (either component) is also shown. Left: Typical viewing geometry for the case $\theta_{j} \gg \Gamma^{-1}$; the wavevector $\boldsymbol{k}$ lies outside the plane of the shock. Right: Viewing geometry for the case $\theta_{j} \sim \Gamma^{-1}$; now $\boldsymbol{k}$ lies roughly in the shock plane. 
TABLE 1

Effect of Fireball, Observing, and Field Geometry on the Polarization

\begin{tabular}{|c|c|c|c|c|c|}
\hline $\begin{array}{l}\text { Fireball } \\
\text { GEOMETRY }\end{array}$ & $\begin{array}{c}\text { SHOck } \\
\text { EMISSION }^{\mathrm{a}}\end{array}$ & \multicolumn{2}{|c|}{ UNIFORM FIELD } & \multicolumn{2}{|c|}{ RANDOM FIELD } \\
\hline \multirow[t]{2}{*}{$\theta_{j} \gg \Gamma^{-1}$} & & $\begin{array}{l}\qquad f>h \\
\text { Faraday depolarization }\end{array}$ & $\begin{array}{l}f f>h \\
\text { Faraday depolarization }\end{array}$ & \multicolumn{2}{|c|}{$\begin{array}{c}|f|>|h| \\
\text { no propagation effects }\end{array}$} \\
\hline & FS & $\Pi_{C} \gg \Pi_{L}$ at $\nu<1 \mathrm{GHz}$ & $\Pi_{C} \gg \Pi_{L}$ at $\nu<1 \mathrm{GHz}$ & no polarization & $\Pi \lesssim 10 \%$ \\
\hline$\theta_{j} \sim \Gamma^{-1} \ldots \ldots \ldots \ldots \ldots \ldots$ & $\mathrm{RS}$ & $\begin{aligned} \Pi_{C} \gg \Pi_{L} \text { at } \nu & <\text { few } \times 10^{14} \mathrm{~Hz} \\
h & \gg f\end{aligned}$ & $\begin{array}{c}\Pi_{C} \gg \Pi_{L} \text { at } \nu<\text { few } \times 10^{14} \mathrm{~Hz} \\
f>h\end{array}$ & $\begin{array}{l}\text { no polarization } \\
\qquad|h| \gg|f|\end{array}$ & $\begin{array}{l}\Pi \lesssim 10 \% \\
|f|>|h|\end{array}$ \\
\hline
\end{tabular}

\footnotetext{
${ }^{\text {a }}$ FS: Forward shock emission; RS: reverse shock emission.

b Field normal to plane of shock.

c Field parallel to plane of shock.
}

effects, and hence in qualitatively different polarization properties of the emitted radiation, as we explain next.

In $\S 4.2$ we show that the frequency ranges where observational fingerprints of plasma propagation effects are expected are $\nu \sim 1 \mathrm{GHz}$ for the forward-shock emission, and $\nu \sim 10^{13} \mathrm{~Hz}$ for the reverse-shock emission. Let us assume that the plasma is permeated by a large-scale magnetic field, and denote by $R_{f / h}=(f / h) \sin ^{2} \vartheta / \cos \vartheta$ the (geometry-independent) ratio of the propagation coefficients in the relevant frequency ranges. The plasma parameters we derive in $\S 4.2$ imply that $R_{f / h} \sim 10(\nu / 1 \mathrm{GHz})^{-1 / 3}$ in the forward shock, and $R_{f / h} \sim$ $10^{3}\left(\nu / 10^{13} \mathrm{~Hz}\right)$ in the reverse shock. ${ }^{5}$ Consider now a typical jet with $\theta_{j} \gg \Gamma^{-1}$. The high $R_{f / h}$ imply that whatever the direction of the magnetic field, in order for $h$ to dominate over $f$ (i.e., in order for $\vartheta$ to be sufficiently close to $\pi / 2$ ), $\boldsymbol{k}$ must lie within a very narrow solid angle. Consequently, a configuration in which $f \gg h$ is much more probable than one in which $h \gg f$ (by a factor of $\sim R_{f / h}-1 \sim 9$ in the forward or $\sim 10^{3}$ in the reverse shocks). Next, consider a narrow jet observed slightly off-axis, with $\theta_{j} \sim \Gamma^{-1}$. Recall that in this regime, the vector $\boldsymbol{k}$ is lying in the shock plane (see Fig. 2, right). If the uniform magnetic field also lies predominantly in the shock plane, we find again that $f \gg h$ is much more probable than $h \gg f$ (by factors of $\sim 7$ and $\sim 10^{2.5}$ for the forward and reverse shocks, respectively). If, however, $\boldsymbol{B}$ is normal to the shock plane, it is necessarily also normal to $\boldsymbol{k}$, and therefore $h \gg f$. The normal modes of propagating radiation are then linearly polarized, and the resulting emission has markedly different polarization properties.

In the next section we calculate propagation effects in both geometric regimes. The case $f \gg h$ is studied in more detail, since this is the characteristic case for a typical GRB jet. The polarization pattern for this regime is shown in Figure 5. In Figure 6 we show the polarization properties of the $h \gg f$ regime, which, as we argued above, is probable only for a narrow jet observed off-axis, with a uniform magnetic field normal to the shock plane. The qualitative results of this discussion,

\footnotetext{
${ }^{5}$ The reason for the much larger value of $R_{f / h}$ in the reverse shock is essentially the fact that reverse-shock radiation travels through a thick slab of cold plasma, and so the values of $f$ and $h$ follow from eq. (4) rather than from eqs. (5) and (6), as they do in the forward shock. The values we give here for $R_{f / h}$ are obtained from plasma parameters of a fireball expanding into a uniform-density ISM. Nevertheless, the corresponding values for a fireball expanding into a wind are similar, and hence this scenario will not be treated separately in this section.
}

complemented by some quantitative consequences of the actual calculations, are summarized in Table 1.

\section{APPLICATION TO GRB AFTERGLOWS}

The application of the results derived in $\S 2$ to plasmas of GRB afterglows requires knowledge of the plasma number density, strength and structure of the magnetic field, and the electron distribution function produced by the shock. These parameters, in turn, depend on the total energy $E$ carried by the relativistic plasma, the number density $n$ of the ambient gas, the duration $T$ of the burst, the Lorentz factor $\Gamma_{i}$ reached by the outflow prior to the deceleration phase, the fractions $\epsilon_{e}$ and $\epsilon_{B}$ of the energy density carried by relativistic electrons and magnetic field, respectively, and the spectral index $p=$ $-d \ln n_{e} / d \ln \gamma_{e}$ of the assumed power-law distribution of the shock-accelerated electrons. When considering a fireball expanding into a wind-stratified medium, $n$ is replaced by $\dot{M} / 4 \pi m_{p} R^{2} v_{w}$, involving the ratio between the progenitor's mass-loss rate, $\dot{M}$, and the wind velocity, $v_{w}$ (where $m_{p}$ is the mean atomic mass for the gas). We therefore briefly address the observational constraints on these parameters, on which our choices of parameter values is based.

We begin with the parameters characterizing the explosion. First, we focus on the subclass of long-duration $(T \gtrsim 2 \mathrm{~s})$ GRBs, since these are the only bursts localized by the BeppoSAX and HETE-2 missions with identified afterglows. We therefore express our results in units of $T_{1}=T / 10 \mathrm{~s}$.

Second, as we are interested in the emission prior to the jet break time, the energy scale determining the dynamics is not the actual explosion energy, but rather its isotropic equivalent, i.e., the total energy derived assuming isotropic emission. Examination of the list of all bursts with measured redshifts indicates $E_{\gamma, \text { iso }} \simeq 3 \times 10^{53}$ ergs as the mean isotropic equivalent energy inferred from the $\gamma$-ray fluence (Bloom et al. 2003). Since this value must be smaller than the total kinetic energy carried by the plasma, it is reasonable to express $E_{\text {iso }}$ in units of $10^{54}$ ergs. Hereafter we drop the subscript "iso" for brevity.

Considering now the ambient gas density, when the observed light curve can be reconciled with expansion into a uniform density ISM, Bloom et al. (2003) find $n$ to typically lie in the range $10^{0.5 \pm 1} \mathrm{~cm}^{-3}$. We therefore adopt a parametrization with $n_{0}=n /\left(1 \mathrm{~cm}^{-3}\right)$. On the other hand, it is now commonly believed that the long-duration GRBs are probably associated with core-collapse supernovae. This association was originally motivated by evidence of intense star formation in identified GRB host galaxies (Bloom et al. 2002), and by additional 
evidence for optical supernovae emission in several GRB afterglows (Bloom 2004). Finally, the SN-GRB relation was put on solid grounds with the recent detection of a supernova emission spectrum (SN 2003dh) associated with GRB 030329 (Stanek et al. 2003; Hjorth et al. 2003). Considering a likely phase of mass loss during the late stages of the massive star's life, it is natural to examine a scenario in which the fireball expands into a wind medium. Below we consider the simplest wind model, in which prior to the explosion the progenitor star ejects mass at a constant rate $\dot{M}=10^{-5} \dot{M}_{-5} M_{\odot} \mathrm{yr}^{-1}$ and at a constant speed $v_{w}=10^{3} v_{3} \mathrm{~km} \mathrm{~s}^{-1}$ (Chevalier \& Li 1999). These parameters agree with observations of massive stars that are believed to be the progenitors of $\mathrm{SNe} \mathrm{Ib} / \mathrm{c}$ associated with GRBs (Willis 1991). Note that since $n \sim 1 \mathrm{~cm}^{-3}$ for a uniformdensity circumburst gas is inferred from observations carried typically $\sim 10 \mathrm{hr}$ after the burst, this is also consistent with a wind environment, which should have a comparable density at the radius reached by the fireball on a $\sim 10 \mathrm{hr}$ timescale (Livio \& Waxman 2000). Finally, the Lorentz factor reached by the outflow prior to its deceleration by the circumburst gas is typically a few hundred. We choose to parameterize our results in units of $\Gamma_{i, 2.5}=\Gamma_{i} / 10^{2.5}$.

We now turn to consider the parameters $p, \epsilon_{e}$, and $\epsilon_{B}$. There is strong indication that the $p$ and $\epsilon_{e}$ are uniform among different bursts, with $\epsilon_{e}$ close to an equipartition value (Frail et al. 2001; Freedman \& Waxman 2001; Berger et al. 2003), and $p=$ $2.2 \pm 0.1$ (Waxman 1997a; Galama et al. 1998; Frail et al. 2000; Freedman \& Waxman 2001). We therefore adopt $\epsilon_{e}=$ $0.1 \epsilon_{e,-1}$ and $p=2.2$ in the numerical examples we consider below. The value of $\epsilon_{B}$ is less constrained by observations, and its estimated value ranges from $\epsilon_{B} \sim 10^{-1}$ (e.g., Waxman 1997a; Wijers \& Galama 1999) to $\epsilon_{B} \sim 10^{-6}$ (e.g., Wijers \& Galama 1999; Chevalier \& Li 1999; Galama et al. 1998; Waxman \& Loeb 1999). Since in cases where $\epsilon_{B}$ can be reliably constrained by multiwaveband spectra, values close to equipartition are inferred (Frail et al. 2000), we first explore consequences of propagation effects on emission from plasmas with $\epsilon_{B}=0.1 \epsilon_{B,-1}$. This is also the parametrization used in $\S$ 4.1. We then study in $\S 4.4$ the implications of a weak magnetic field, adopting $\epsilon_{B}=10^{-4}$ as an illustrative example.

Next we explore how the propagation effects described in the previous section manifest themselves in the context of a GRB afterglow. In $\S 4.1$ we derive the relevant plasma conditions at the onset of fireball deceleration, considering expansion both into a uniform-density ISM ( $\S 4.1 .1)$ and into a wind medium ( $\S 4.1 .2$ ). In $\S 4.2$ we examine the observable consequences of our derivations, investigating first the case of a uniform, close to equipartition magnetic field. Since a typical GRB jet is characterized by $\theta_{j} \gg \Gamma^{-1}$, implying typically $f \gg h$ in the frequency ranges of interest (see $\S 3$ ), this section gives detailed analysis of this regime. Section 4.3 studies the complementary case, where $h \gg f$. The effect of a weak field, as well as the effect of an entangled field geometry, are treated in $\S$ 4.4. Throughout the rest of this paper, we use primed quantities to denote values measured in the frame that is comoving with the plasma.

\subsection{Plasma Parameters during the Early Afterglow}

\subsubsection{Expansion into a Uniform-Density ISM}

The relativistic blast wave driven into the ambient gas by the energetic explosion approaches a self-similar behavior (Blandford \& McKee 1976) once a reverse shock crosses the ejecta and heats it (Mészáros \& Rees 1997). During this transition episode both the shocked ambient gas and the heated ejecta propagate with a Lorentz factor close to that given by the Blandford-McKee self-similar solution (see, e.g. Waxman \& Draine 2000),

$$
\Gamma^{(R, F)} \simeq\left(\frac{17 E}{1024 \pi n m_{p} c^{5} T^{3}}\right)^{1 / 8} \simeq 328 E_{54}^{1 / 8} n_{0}^{-1 / 8} T_{1}^{-3 / 8}
$$

where $E_{54}=E / 10^{54}$ ergs is the isotropic-equivalent of the fireball energy, $T_{1}=T / 10 \mathrm{~s}$ is the burst duration, and $n_{0}=$ $n / 1 \mathrm{~cm}^{-3}$ is the ambient number density. The superscripts $R, F$ denote the reverse and forward shocked plasmas, respectively. The corresponding electron number densities are accordingly given by the shock jump conditions,

$$
\begin{aligned}
n_{e}^{\prime(F)} & =4 \Gamma n \simeq 1300 E_{54}^{1 / 8} n_{0}^{7 / 8} T_{1}^{-3 / 8} \mathrm{~cm}^{-3} \\
n_{e}^{\prime(R)} & \simeq\left(\Gamma^{2} / \Gamma_{i}\right) n_{e}^{\prime(F)} \\
& \simeq 3.99 \times 10^{5} E_{54}^{3 / 8} n_{0}^{5 / 8} T_{1}^{-9 / 8} \Gamma_{i, 2.5}^{-1} \mathrm{~cm}^{-3} .
\end{aligned}
$$

The comoving widths of shocked forward and reverse plasma shells can be shown to be comparable, and are estimated as

$$
W^{\prime(F, R)} \simeq R / 4 \Gamma \simeq 9.8 \times 10^{13} E_{54}^{1 / 8} n_{0}^{-1 / 8} T_{1}^{5 / 8} \mathrm{~cm},
$$

where $R \simeq 1.3 \times 10^{17} \mathrm{~cm}$ is the radius at which the reverse shock is propagating through the ejecta. From the shock jump conditions we infer the strength of the magnetic field,

$B^{\prime(F, R)}=\left(32 \pi \epsilon_{B} n m_{p} c^{2}\right)^{1 / 2} \Gamma \simeq 40 E_{54}^{1 / 8} n_{0}^{3 / 8} T_{1}^{-3 / 8} \epsilon_{B,-1}^{1 / 2} \mathrm{G}$.

The distribution function of relativistic electrons injected by the shocks is assumed to increase as $\gamma_{e}^{2}$ up to a characteristic Lorentz factor $\gamma_{m}$ (Gruzinov \& Waxman 1999), and decrease as a power law $\gamma_{e}^{-p}$ (with $p=2.2$ ) at higher values of the Lorentz factor. Then the jump conditions imply

$$
\begin{aligned}
& \gamma_{m}^{(F)}=\frac{4(p-2)}{3(p-1)} \epsilon_{e} \frac{m_{p}}{m_{e}} \Gamma \simeq 1.32 \times 10^{4} E_{54}^{1 / 8} n_{0}^{-1 / 8} T_{1}^{-3 / 8} \epsilon_{e,-1}, \\
& \gamma_{m}^{\prime(R)}=\frac{4(p-2)}{3(p-1)} \epsilon_{e} \frac{m_{p}}{m_{e}} \frac{\Gamma_{i}}{\Gamma} \simeq 43 E_{54}^{-1 / 8} n_{0}^{1 / 8} T_{1}^{3 / 8} \Gamma_{i, 2.5} \epsilon_{e,-1} .
\end{aligned}
$$

The observed characteristic synchrotron frequencies corresponding to the Lorentz factor of the thermal electrons in these two shock scenarios are

$$
\begin{aligned}
& \nu_{m}^{(F)} \simeq 9.61 \times 10^{18} E_{54}^{1 / 2} T_{1}^{-3 / 2} \epsilon_{e,-1}^{2} \epsilon_{B,-1}^{1 / 2} \mathrm{~Hz} \\
& \nu_{m}^{(R)} \simeq 1.04 \times 10^{14} n_{0}^{1 / 2} \Gamma_{i, 2.5}^{2} \epsilon_{e,-1}^{2} \epsilon_{B,-1}^{1 / 2} \mathrm{~Hz}
\end{aligned}
$$

In the parlance of the high-energy and IR communities, respectively, these read an energy $E_{\mathrm{ph} . m}^{(F)}=39.7 \mathrm{keV}$ for the forward shock, and a wavelength $\lambda_{m}^{(R)} \stackrel{\mathrm{ph}, m}{=} 2.9 \mu \mathrm{m}$ for the reverse shock.

\subsubsection{Expansion into a Wind}

We now examine a fireball expanding into a wind. The simple wind model considered here results in a nonhomogeneous ambient gas with a profile $n \propto R^{-2}$. The reverse shock crosses the ejecta at a typical radius of $2.2 \times 10^{16} \mathrm{~cm}$, at which 
time the plasmas shocked by the forward and reverse shocks propagate together with a Lorentz factor

$$
\Gamma^{(F, R)} \simeq 135 E_{54}^{1 / 4}\left(\dot{M}_{-5} / v_{3}\right)^{-1 / 4} T_{1}^{-1 / 4} .
$$

Following the reasoning employed above, we obtain the other relevant plasma parameters for the wind scenario:

$$
\begin{aligned}
W^{\prime(F, R)} & \simeq 4.04 \times 10^{13} E_{54}^{1 / 4}\left(\dot{M}_{-5} / v_{3}\right)^{-1 / 4} T_{1}^{3 / 4} \mathrm{~cm} \\
n_{e}^{\prime(F)} & \simeq 3.43 \times 10^{5} E_{54}^{-3 / 4}\left(\dot{M}_{-5} / v_{3}\right)^{7 / 4} T_{1}^{-5 / 4} \mathrm{~cm}^{-3} \\
n_{e}^{\prime(R)} & \simeq 1.78 \times 10^{7} E_{54}^{-1 / 4}\left(\dot{M}_{-5} / v_{3}\right)^{5 / 4} T_{1}^{-7 / 4} \Gamma_{i, 2.5}^{-1} \mathrm{~cm}^{-3} \\
B^{\prime(F, R)} & \simeq 418 E_{54}^{-1 / 4}\left(\dot{M}_{-5} / v_{3}\right)^{3 / 4} T_{1}^{-3 / 4} \epsilon_{B,-1}^{1 / 2} \mathrm{G} \\
\gamma_{m}^{(F)} & \simeq 5440 E_{54}^{1 / 4}\left(\dot{M}_{-5} / v_{3}\right)^{-1 / 4} T_{1}^{-1 / 4} \epsilon_{e,-1} \\
\gamma_{m}^{\prime(R)} & \simeq 105 E_{54}^{-1 / 4}\left(\dot{M}_{-5} / v_{3}\right)^{1 / 4} T_{1}^{1 / 4} \Gamma_{i, 2.5} \epsilon_{e,-1} .
\end{aligned}
$$

The characteristic synchrotron frequencies in the wind scenario are

$\nu_{m}^{(F)} \simeq 7.0 \times 10^{18} E_{54}^{1 / 2} T_{1}^{-3 / 2} \epsilon_{e,-1}^{2} \epsilon_{B,-1}^{1 / 2} \mathrm{~Hz}$,

$\nu_{m}^{(R)} \simeq 2.60 \times 10^{15} E_{53}^{-1 / 2}\left(\dot{M}_{-5} / v_{3}\right) T_{1}^{-1 / 2} \Gamma_{i, 2.5}^{2} \epsilon_{e,-1}^{2} \epsilon_{B,-1}^{1 / 2} \mathrm{~Hz}$.

\subsection{Observational Consequences for a Uniform, Close to Equipartition Magnetic Field: The "Typical Jet" Regime}

The formalism developed in $\S 2.1$ was applied to the plasma conditions derived above. We assumed that the magnetic field and electron density are uniform across the slab. As an electron drifts away from the shock front with a comoving velocity $c / 3$, it loses energy through synchrotron emission. Consider, for simplicity, an isotropic, monoenergetic electron population, with a Lorentz factor $\gamma_{i}^{\prime}$ endowed by the shock. Assuming that this population remains isotropic (in momentum space of the comoving plasma) as it loses energy to radiation, the mean Lorentz factor decreases as

$$
\gamma^{\prime}\left(z^{\prime}\right)=\frac{\gamma_{i}^{\prime}}{1+3 \sigma_{\mathrm{T}} B^{\prime 2} \gamma_{i}^{\prime} z^{\prime} / 6 \pi m_{e} c^{2}},
$$

where $z^{\prime}$ is the comoving separation from the shock front. Assuming now that the shock injects a spectrum of electron energies, equation (17) implies that the shape of the energy distribution function is modified as the separation from the shock front increases. As a result, only a thin slab of width $3 \times 10^{11} \mathrm{~cm}$ behind the forward shock is dominated by a relativistic electron population, while the reverse shock front is separated from the observer by a cold slab $2 \times 10^{13} \mathrm{~cm}$ wide (for a fireball expanding into ISM; the corresponding widths for the wind scenario are $5 \times 10^{9} \mathrm{~cm}$ and $4 \times 10^{13} \mathrm{~cm}$, respectively). As this strongly affects the values of emission, absorption and propagation coefficients (see Appendix), we integrated the radiative transfer equation (2) by dividing the emitting slab into a large number of subslabs, such that within each subslab the electron energy spectrum did not change significantly. The coefficients of emission, absorption, and propagation could then be assumed to be constant within each subslab, allowing an analytic solution of the transfer equation. This division was refined until convergence was obtained.

Equations (4), (5), and (6) imply a strong dependence of the propagation coefficients on $\vartheta$, the comoving angle between the
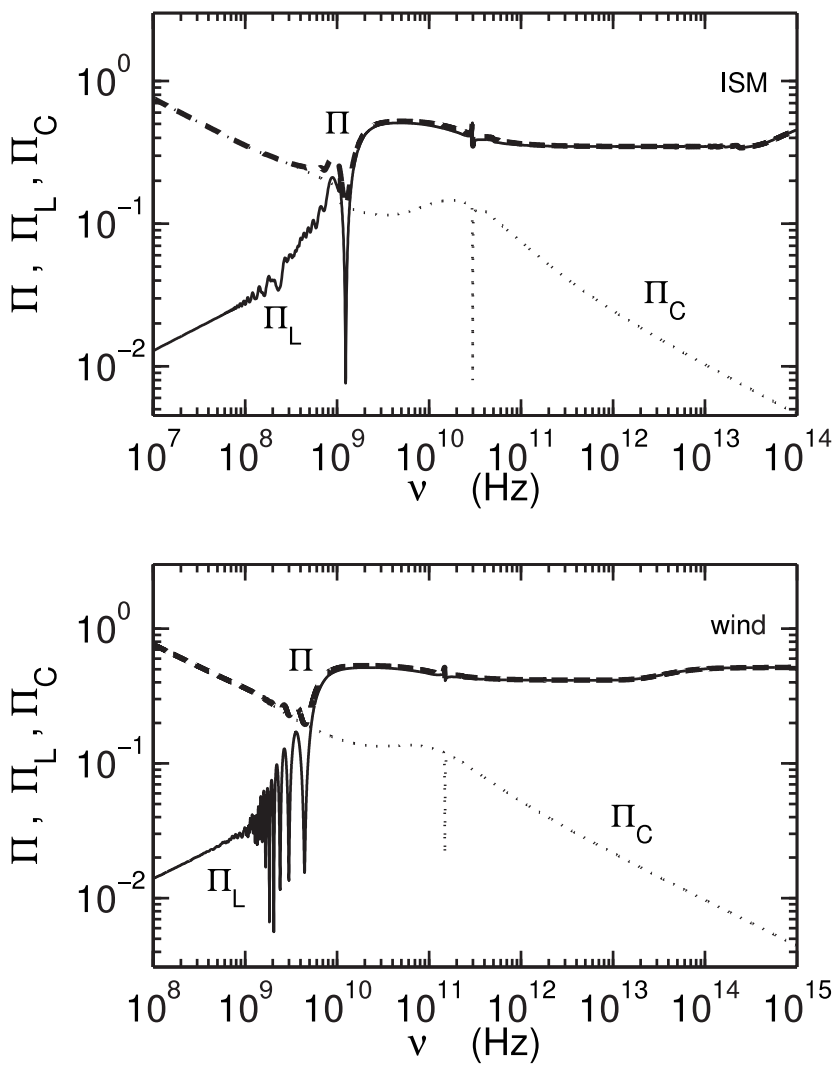

FIG. 3.-Propagation effects on synchrotron radiation propagating in a magnetoactive plasma. The plasma conditions are characteristic of forward shocks for a fireball expanding into a uniform-density ISM (top) and into wind (bottom), during the onset of fireball deceleration. The magnetic field is assumed to be uniform and close to equipartition $\left(\epsilon_{B}=10^{-1}\right)$. Both figures show the degrees of linear polarization $\Pi_{L}$ (solid line), circular polarization $\Pi_{C}$ (dotted line), and the total degree of polarization $\Pi$ (dashed line).

directions of the wavevector and the magnetic field. Yet, as argued in $\S 3$, the observing geometry for a typical GRB jet are likely to give rise to a situation in which $f \gg h$. In such circumstances $\vartheta \sim \pi / 2$ is most unlikely, and the angular dependence of $f$ and $h$ may be ignored, for simplicity. This simplification was adopted in the calculation descried below, and gives a good order-of-magnitude estimate of the emitted polarization of a typical jet with probable viewing geometry. The case $h \gg f$, occurring when the jet is narrow $\left(\theta_{j} \sim \Gamma^{-1}\right)$ and the magnetic field is predominantly normal to the shock plane (see $\S 3)$, is treated separately in $\S 4.3$.

We verified that all elements of the tensor $\kappa_{i j}$ at all relevant frequencies are much smaller than unity, thus self-consistently justifying the analysis of $\S 2.1$, which assumed that the electric field is perpendicular to the wavevector.

The resulting emitted linear, circular, and total degrees of polarization at the onset of fireball deceleration are shown in Figure 3 (forward shock) and Figure 4 (reverse shock). We show results for both ISM and wind scenarios. All four graphs exhibit broadly the same behavior. Because of the Faraday effect, the linear polarization of emitted radiation is strongly suppressed at low frequencies, and circular polarization dominates. At a frequency of $\sim 1 \mathrm{GHz}(30 \mathrm{GHz})$ in the forward shock into ISM (wind), and $\sim 3 \times 10^{13} \mathrm{~Hz}$ in the two reverse shocks, linear polarization takes over. At the transition frequency the total degree of polarization has a global minimum (10\%-20\%). Only at frequencies much higher than this transition frequency 

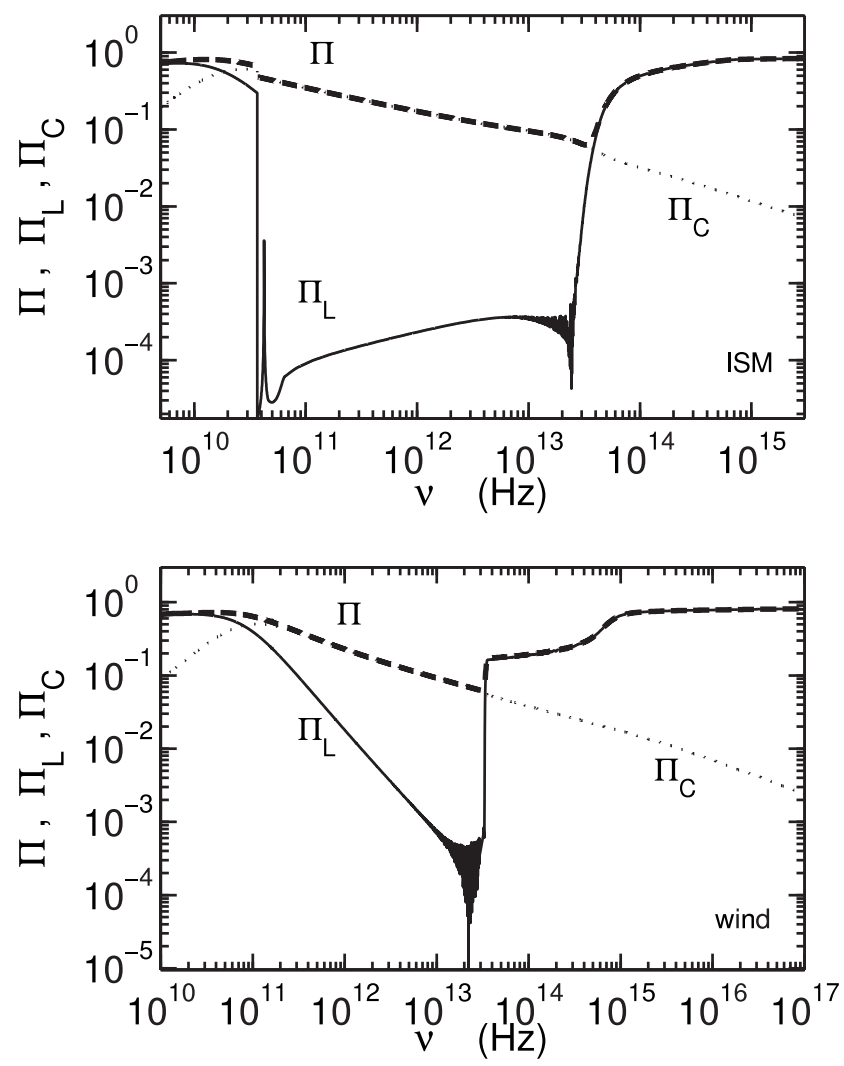

FIG. 4.-Propagation effects on synchrotron radiation propagating in a magnetoactive plasma. The plasma conditions are characteristic of reverse shocks for a fireball expanding into a uniform-density ISM (top) and into wind (bottom), during the onset of fireball deceleration. The magnetic field is assumed to be uniform and close to equipartition $\left(\epsilon_{B}=10^{-1}\right)$. Both figures show the degrees of linear polarization $\Pi_{L}$ (solid line), circular polarization $\Pi_{C}$ (dotted line), and the total degree of polarization $\Pi$ (dashed line).

is the canonical degree of polarization (dominated by its linear component) reached.

The emission from the reverse shock overtakes the forwardshock emission at frequencies above $\nu \sim 2 \times 10^{10} \mathrm{~Hz}$. We find that for the reverse shock, the transition from circular to linear polarization is accompanied by a strong oscillation of the polarization position angle as function of frequency. Because of synchrotron losses, most of the plasma heated by the reverse shock cools quickly on a dynamic timescale, and consequently Faraday rotation by the cold plasma strongly affects radiation emitted from regions closer to the reverse shock front. The resulting oscillation is a hallmark of reverse-shock emission up to UV frequencies $\left(10^{15}-10^{16} \mathrm{~Hz}\right)$, and is characterized by $\Delta \nu / \nu \simeq 10^{-1} \nu_{15}^{2}$ (from eq. [7]), where $\nu_{15}$ is the observed frequency in units of $10^{15} \mathrm{~Hz}$; Observation of these oscillations may therefore be limited by instrument resolution in the optical and particularly the IR bands. Note also that during the transition to self-similar dynamics, emission is observed from an area on the sky with a radius comparable to $R / \Gamma$ (Waxman 1997b). Hence, radiation at observed frequency $\nu$ spans a range of comoving frequencies $\Delta \nu^{\prime}$. Since radiation at different comoving frequencies is subject to different phase shifts between normal modes, spectropolarimetric features characterized by a smaller comoving $\Delta \nu^{\prime}$ may be washed out altogether. Although an exact, quantitative statement regarding the implications for the observability of the Faraday oscillations is beyond the scope of this paper, we expect that the depolarization

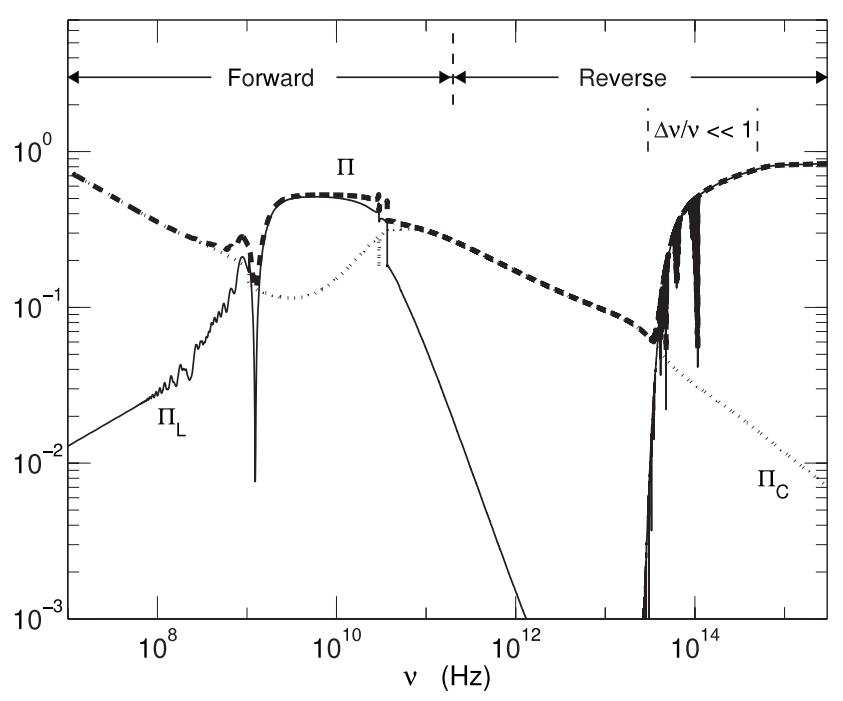

FIG. 5.-Polarization pattern of combined (superposed) reverse- and forward-shock emission, for a fireball expanding into a uniform-density ISM. Shown are the degrees of linear polarization $\Pi_{L}$ (solid line), circular polarization $\Pi_{C}$ (dotted line), and the total degree of polarization $\Pi$ (dashed line). At frequencies $\nu<2 \times 10^{11} \mathrm{~Hz}$, the polarization is determined by forward-shock emission, whereas above that frequency it is dominated by the emission from the reverse shock. In the frequency range $3 \times 10^{13} \mathrm{~Hz}<\nu \lesssim$ few $\times 10^{14} \mathrm{~Hz}$ marked by $\Delta \nu \ll \nu$ in the figure, rapid oscillations of the polarization position angle may not be resolved, thus suppressing linear polarization, rendering circular polarization dominant. The calculations leading to this result correspond to a typical GRB jet with an opening angle $\theta_{j} \gg \Gamma^{-1}$, implying $f \gg h$ (see $\S 3)$.

of the linear component, caused by either low spectral resolution or smearing of the signal by the source plasma, is expected to yield a signal dominated by a circular polarization $\Pi_{C} \sim 0.1\left(\nu / 10^{13} \mathrm{~Hz}\right)^{-1 / 3}$, surviving up to UV frequencies.

A superposition of the polarization patterns of the forward and reverse shocks is shown in Figure 5, for the ISM scenario. The polarization is dominated by the reverse-shock emission at frequencies above $\sim 2 \times 10^{11} \mathrm{~Hz}$. The analogous superposition of forward- and reverse-shock polarization for the wind scenario is qualitatively similar.

Remarkably, the main characteristics of the polarization pattern, including the frequency of transition from circular to linear polarization, are largely independent of the circumburst density, and are dictated predominantly by the nature of the shock (i.e., whether the shock is forward or reverse). This is particularly intriguing in view of the 3 orders of magnitude factor separating the densities of ambient gas in the ISM and wind scenarios, at the onset of fireball deceleration. Considering the two forward shocks first, it is straightforward to show that in both cases $\nu_{a}^{\prime} \ll \nu_{m}^{\prime}$, where $\nu_{a}^{\prime}$ is the (comoving) synchrotron self-absorption frequency. At smaller frequencies, $\nu^{\prime}<\nu_{a}^{\prime}$, the effective width $W_{\text {eff }}^{\prime}\left(\nu^{\prime}\right)$ from which photons are emitted scales as $W_{\text {eff }}^{\prime}\left(\nu^{\prime}\right) \propto\left[\kappa^{\prime}\left(\nu^{\prime}\right)\right]^{-1} \propto \nu^{\prime 5 / 3}$. By virtue of equations (3) and (5), then, we have at low frequencies $\Pi_{L}\left(\nu^{\prime}\right) \propto W_{\text {eff }}^{\prime} \nu^{\prime 2} \propto \nu^{\prime 1 / 3}$. On the other hand, the degree of circular polarization at frequencies $\nu^{\prime} \gg \nu_{B}^{\prime}$ scales as $\nu^{\prime-1 / 3}$. Denoting the frequency of the transition from circular to linear polarization by $\nu_{t}$, we therefore find $\nu_{t}^{\prime} \propto\left(\nu_{B}^{\prime} \nu_{a}^{\prime}\right)^{1 / 2}$. Transforming to the observer's frame, we find for the forward shock in the ISM, $\nu_{t} \propto n^{27 / 80} \sim n^{1 / 3}$. Substituting here the ambient density for the wind scenario at the transition-phase radius, which is larger by $\sim 3$ orders of magnitude, and correcting for the much narrower slab, which effectively emits photons in this 
scenario (because of the more efficient synchrotron cooling), we obtain $\nu_{t}$ in the wind scenario larger by a factor of $\sim 3$, as can be readily verified in Figure 3. Considering now the two reverse shocks, the frequency at which linear polarization takes over circular polarization is simply the synchrotron selfabsorption frequency, since at $\nu>\nu_{a}$ the region closest to the shock front becomes observable. As this region is populated by electrons that are still highly relativistic, it contributes most of the photons above $\nu_{a}$, while the thick slab of cold plasma separating this emitting region from the observer is characterized by a much smaller emissivity. Radiation emerging from the emitting region close to the reverse shock front is subject to a large Faraday rotation owing to the presence of the cold plasma; however, since the emissivity of the cold plasma is small, this has only a minor effect on the degree of linear polarization of that radiation. The similar $\nu_{t}$ in the two reverse shock scenarios is thus a consequence of a similar selfabsorption frequency in these two cases.

We note that our calculations indicate that the plasma shocked by the forward shock does not affect the polarization properties of the emission from the reverse shock, since it is optically thin in the frequency range where the reverse shock emission exhibits interesting polarization features. Moreover, only the nonrelativistic component of the plasma behind the forward shock introduces a Faraday rotation with a large enough amplitude above a frequency of $10^{13} \mathrm{~Hz}$. Yet this plasma contributes only negligibly to the emission and absorption of radiation, and hence does not change the linear, circular, or total degrees of polarization, but merely weakly modifies the pattern of position angle oscillations.

\subsection{Observational Consequences: The "Narrow Jet" Regime}

A narrow jet $\left(\theta_{j} \sim \Gamma^{-1}\right)$ observed slightly off-axis with a uniform magnetic field normal to the plane of the shock(s) is the only geometric configuration in which the propagation coefficient $h$ is very likely to dominate over $f$ (see $\S 3$ ). We repeated the calculations of plasma propagation effects on the polarization properties of early afterglow emission, assuming that uniform, close to equipartition fields $\left(\epsilon_{B}=0.1\right)$ permeate the reverse and forward shocked plasmas, but this time neglecting $f$ in comparison to $h$ (i.e., setting $\vartheta=\pi / 2$ in eqs. [4]-[6]). The resulting linear, circular, and total degrees of polarization of the combined forward-reverse shock emission are shown in Figure 6 . The emerging picture is markedly different from the one described above ( $\S 4$, Fig. 5). Linear polarization is not suppressed by Faraday rotation, and dominates over circular polarization in the entire frequency range of interest. (Notice, however, the comparable degree of circular polarization in the emission from the forward shock at low frequencies, $\nu \lesssim$ few $\times 100 \mathrm{MHz}$, due entirely to the intrinsic circular polarization of synchrotron at low frequencies.) In addition, we notice the absence of Faraday oscillations of the polarization position angle $\chi$ at high (IR-UV) frequencies. Not being ironed out by the oscillations, linear polarization is therefore expected to prevail in this case in the IR-UV range as well, in contrast to the situation in the case of a typical jet. Interestingly, linear polarization is suppressed in the microwave and IR band (contributed solely by reverse-shock emission) not by the action of plasma effects, but rather by the suppression of the Stokes parameter $Q$ relative to $I$ at large optical depths. ${ }^{6}$

\footnotetext{
${ }^{6}$ See, e.g., Pacholczyk (1977) for an analogous suppression of $\Pi_{L}$ at $\nu \gg \nu_{m}$
}

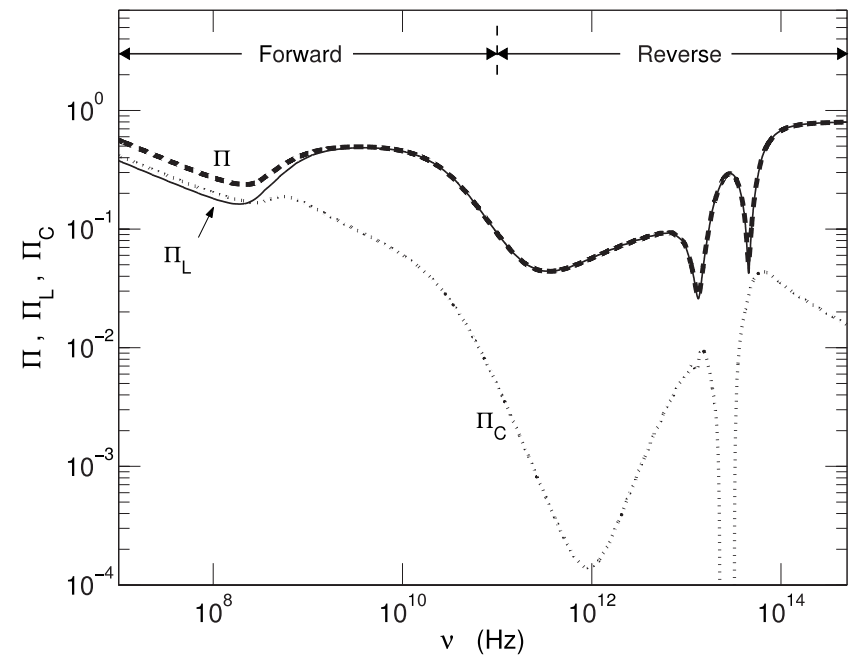

FIG. 6.- Same as Fig. 5, but with $\vartheta$ set to $\pi / 2$, implying effectively $h \gg f$. This situation is atypical, and is probable for a narrow jet of an opening angle $\theta_{j} \sim \Gamma^{-1}$, with the jet observed off-axis and the uniform magnetic field directed normal to the shock plane. Plasma parameters correspond to a fireball expanding into the ISM. However, we find that the picture is also qualitatively similar in the wind scenario.

\subsection{Implications of Magnetic Field Strength and Structure}

As mentioned above, the fraction of the postshock energy density carried by the magnetic field, $\epsilon_{B}$, is one of the model parameters least constrained by observations. Since the propagation effects addressed in this paper are particularly sensitive to the strength of the magnetic field, it is natural to explore how the polarization of emitted radiation depends on the amplitude of $B$. We therefore consider $\epsilon_{B}=10^{-4}$ as a fiducial value for the weak-field regime, keeping all other fireball parameters unchanged.

We find that the general features exhibited by the linear, circular, and total degrees of polarization are broadly similar to those described above for the uniform equipartition field scenario. In particular, we find that the linear polarization is suppressed at low frequencies. There are some marked differences, however: the transition frequency from circular to linear polarization is decreased by approximately an order of magnitude compared to the strong-field scenarios described above. In addition, the oscillation pattern of the position angle in the reverse-shock scenario does not survive the decrease in $B$, and is absent in the weak-field regime.

In the case in which the structure of the magnetic field is entangled over length scales $\ell$ much shorter than the typical width of the emitting slabs $W$, the fluctuating phase accumulated between the two normal modes averages to zero, and its rms is suppressed by a factor $(\ell / W)^{1 / 2} \ll 1$, resulting in the absence of the propagation effects discussed above. It is helpful in this context to separate the discussion of the two fireball and observation geometric regimes. Addressing first the "typical jet" regime $\left(\theta_{j} \gg \Gamma^{-1}\right)$ in the random-field scenario $(\ell \ll W)$, we conclude that linear polarization is not subject to suppression due to Faraday rotation. Nevertheless, this does not imply a high degree of linear polarization, because the overall orientation of synchrotron radiation emitted from the many different patches averages to zero. If, however, the scale of the coherent patch is limited only by causality and is thus comparable to the width of the emitting slab, viz. $\ell \sim W$ (Gruzinov \& Waxman 1999), Faraday rotation is not completely suppressed, and some suppression of $\Pi_{L}$ with 
respect to $\Pi_{C}$ is expected. The frequencies of transition from circular to linear polarization are not expected to change substantially, although the degrees of circular and linear polarization will be lower by a factor of a few (with a maximal $\Pi$ at the level of $\sim 10 \%$ ), because of the different directions that the magnetic field may assume in the $\sim 50$ coherent, causal patches apparent to the observer (Gruzinov \& Waxman 1999). Turning our attention to the "narrow jet" regime, a random, $\ell \ll W$ field will suppress all propagation effects. In this case, a high degree of linear polarization $\left(\Pi_{L} \sim\right.$ a few $\left.\times 10 \%\right)$ may arise, if the random magnetic field has a dominant (fluctuating) component either normal to the plane of the shock or parallel to it (Waxman 2003b; Nakar et al. 2003). The high polarization stems from the orthogonality of the direction $\boldsymbol{k}$ of observed radiation to the direction of the magnetic field in the frame comoving with the plasma (see right panel of Fig. 2 for an illustration). Independent patches limited by causality, as in Gruzinov \& Waxman (1999), will not suppress propagation effects altogether, and the polarization pattern of the emitted radiation is expected to follow qualitatively either Figure 5 or Figure 6, depending on the dominant component of the fluctuating magnetic field, with maximum levels of $\Pi_{L}$ and $\Pi_{C}$ decreased by a factor of a few. These predictions are summarized in Table 1.

To conclude, then, the polarization of the early afterglow emission provides a robust measure of the field's complexity: a dominant, high degree of circular polarization at $\nu \lesssim 1 \mathrm{GHz}$ and again at few $\times 10^{10} \mathrm{~Hz} \lesssim \nu \lesssim$ few $\times 10^{14} \mathrm{~Hz}$ is a clear signature of a large-scale, coherent field present in the plasma. On the other hand, a high degree of linear polarization may result with high probability in the narrow jet regime either from a large-scale field normal to the shock plane, or from an entangled field, and is therefore less informative as far as field structure is concerned.

\section{CONCLUSION}

Ever since it was realized that the dominant mechanism responsible for the observed radiation from GRB afterglows is synchrotron emission, the spectra and polarization at various stages of fireball expansion were calculated by integration of the relevant emissivities and absorption coefficients over varying conditions in the fireball plasma. Indeed, light curves and spectra are successfully reproduced by such procedures. However, the propagation effects of a magnetized, relativistic plasma on the polarized synchrotron radiation may have significant observable outcomes that had so far not been addressed. In particular, the birefringent nature of the magnetized plasma, leading to a phase shift between the normal propagation modes, which is accumulated on the coherence length scale of the magnetic field and manifested as the Faraday effect, may have significant consequences for the polarization of GRB afterglows. A full treatment of this problem, taking into consideration the central part played by synchrotron cooling (see below), is presented here for the first time.

In this work we generalize results by Sazonov (1969), and calculate the terms of the dielectric susceptibility tensor responsible for the Faraday effect for a plasma with an isotropic, relativistic distribution of electrons. We also derive new simple, analytic expressions for the propagation coefficients in various frequency ranges (see eqs. [5], [6], and Appendix A.1). Similar expressions were derived independently by Matsumiya \& Ioka (2003).

We have applied these results to plasma conditions typical of the early afterglow, where plasma effects on the polarization of propagating synchrotron radiation are significant. We consider both the forward and reverse shocks, and show that if the plasma is permeated by a large-scale magnetic field close to equipartition, the polarization pattern of emitted radiation differs decisively from the naive estimates usually quoted in the literature. We show that for a typical GRB jet, in which $\theta_{j} \gg \Gamma^{-1}$ during the early afterglow, a large-scale field results in a strong suppression of linear polarization at low frequencies, i.e., $\nu \lesssim 3 \mathrm{GHz}\left(\nu<3 \times 10^{13} \mathrm{~Hz}\right)$ in a forward (reverse) shock due to Faraday rotation, and a prevalence of circular polarization. Above these transition frequencies linear polarization takes over, and gradually approaches the canonical value. At the frequencies of transition, the total degree of polarization has a global minimum of $10 \%-20 \%$. The emission from the reverse shock above the characteristic synchrotron frequency exhibits strong, fast oscillations of the polarization position angle as a function of frequency. The oscillations mark the emission up to UV frequencies $\left(10^{15}-10^{16} \mathrm{~Hz}\right)$; since they may be spectrally resolved only above $\sim$ a few $\times 10^{14} \mathrm{~Hz}$, this implies a suppression of linear polarization (and a dominating circular polarization as a result) up to the UV band. These prominent fingerprints of the presence of a large-scale magnetic field in the plasma are rather independent of the environment into which the fireball expands, whether it has a uniform density (ISM) or a wind density profile (see $\S 4.2$ ). The suppression of linear polarization at low frequencies is also shown to characterize the case of a uniform field that is much below the equipartition value. However, in the weak-field regime the frequency of transition from circular to linear polarization decreases by approximately an order of magnitude, and the oscillation pattern of the position angle is absent (whence also the dominance of $\Pi_{C}$ up to the UV band). These propagation effects are expected to vanish if the field is entangled over length scales much smaller than the extent of the emitting plasma.

A large-scale magnetic field is likely to give rise to different dispersive effects on the polarization properties if the jet is narrow $\left(\theta_{j} \sim \Gamma^{-1}\right)$ and observed off-axis, and if in addition the field is predominantly normal to the shock plane. In this case we predict the dominance of linear polarization at all frequencies (with the exception of a comparable circular component at low radio frequencies). However, this is not an exclusive hallmark of a large-scale magnetic field in the narrow, off-axis jet regime, since a high degree of linear polarization may also result if the field is entangled over small length scales, as may have been the case in GRB 021206.

Recently, Matsumiya \& Ioka (2003) treated similar propagation effects in the context of GRB afterglows, neglecting the inhomogeneity of the emitting slabs due to synchrotron losses. As we demonstrated explicitly in $\S 4.2$, cooling has a decisive effect, most importantly on the values of the propagation coefficients. This leads to differences between Matsumiya \& Ioka's results and ours (e.g., the higher degree of circular polarization, by approximately an order of magnitude, that we find in the transition frequencies $1 \mathrm{GHz}$ and few $\times$ $\left.10^{13} \mathrm{~Hz}\right)$.

Our results suggest that spectropolarimetric observations in the radio and IR bands during the early stages of the afterglow may be used as a unique probe of the structure and strength of the magnetic field. A handle on the field configuration is of particular importance in studying the plasma conditions in the reverse shock, since this plasma is essentially the one outflowing from the compact source, and hence may reveal valuable information on the processes driving the outflow and on the nature of 
the progenitor. This technique, complementary to measurements of the $\gamma$-ray polarization, may therefore be powerful in placing stringent constraints on models of the "inner engine" and the origin of the magnetic field, and promote our understanding of the physics of collisionless shock waves.
We thank the anonymous referee for helpful suggestions and instructive comments. This research was supported in part by ISF, AEC, and Minerva grants for E. W., and by grants from NASA (NAG 5-13292) and NSF (AST 00-71019, AST 0204514) for A. L.

\section{APPENDIX A}

\section{PROPAGATION, EMISSION, AND ABSORPTION COEFFICIENTS IN A RELATIVISTIC PLASMA}

\section{A1. PROPAGATION COEFFICIENTS IN A RELATIVISTIC PLASMA}

We briefly review here the derivation of the coefficients $f$ and $h$ (see eqs. [1] and [2]), associated with propagation effects, for the case of a plasma with an isotropic, relativistic electron population. The deviation of the dielectric tensor

$$
\epsilon_{i j}(\omega, \boldsymbol{k})=\delta_{i j}+4 \pi \kappa_{i j}(\omega, \boldsymbol{k})
$$

from its value in vacuum $\left(\delta_{i j}\right)$ is assumed to be small, so that the radiation can be regarded as transverse (Sazonov 1969).

To obtain $f$, a quantity that is first order in $B$, we separate the perturbation to the equilibrium distribution function into two contributions, one that is independent of the magnetic field, and one that is linear in the magnetic field. The Maxwell equations lead to a dispersion relation

$$
c^{2} k^{2} / \omega^{2} E_{i}=\epsilon_{i j} E_{j}, \quad i, j=1,2
$$

which, when coupled to a linearized Vlasov equation, gives the nondiagonal elements

$$
\epsilon_{12}=-\epsilon_{21}=i \frac{\tilde{\omega}_{p}^{2} \tilde{\omega}_{B}}{\omega} \cos \vartheta \int d^{3} p \frac{1}{\left(\omega-k v_{\|}\right)^{2}} \frac{d f^{(0)}}{d p} \frac{p_{\perp 1}^{2}}{\gamma^{2} p}
$$

Here $\tilde{\omega}_{p}^{2}=4 \pi n_{e} e^{2} / m_{e}$ and $\tilde{\omega}_{B}=e B / m_{e} c$ are the nonrelativistic (electron) plasma frequency and Larmor frequency, respectively; $\vartheta$ is the angle between $\boldsymbol{k}$ and $\boldsymbol{B}, \gamma=\left(p^{2}+m_{e}^{2} c^{2}\right)^{1 / 2} / m_{e} c$ is the particle's Lorentz factor, and $f^{(0)}(p)$ is the unperturbed isotropic electron distribution function.

Sagiv \& Waxman (2002) showed numerically that the value of $\epsilon_{12}$ is not very sensitive to the exact shape of the distribution function, and may be approximated by substituting a monoenergetic electron distribution $f^{(0)}(\gamma) \propto \delta\left(\gamma-\gamma_{m}\right)$ in equation (A3), with $\gamma_{m}$ a characteristic electron Lorentz factor (typically at the peak of the distribution function). This leads to a simple expression for the propagation coefficient $f$ in the relativistic regime,

$$
f_{\text {rel }}(\nu) \simeq \frac{e^{3} n_{e} B \cos \vartheta}{\pi m_{e}^{2} c^{2} \nu^{2}} \frac{\ln \gamma_{m}}{\gamma_{m}^{2}}
$$

To obtain $h_{\text {rel }}$, we started with equation (9) of Sazonov (1969), who used a kinetic equation approach to obtain analytic expressions for the elements of the susceptibility tensor $\kappa_{i j}$. Using the notation of the current paper, this equation reads

$$
\kappa_{11}=-\frac{1}{4} \frac{e^{2} m_{e}^{2} c^{6}}{\omega^{2}}\left(\frac{\tilde{\omega}_{B} \sin \vartheta}{\omega}\right)^{2} \int_{0}^{\infty} d \mathcal{E} \frac{d}{d \mathcal{E}}\left[\frac{d n(\mathcal{E}) / d \mathcal{E}}{\mathcal{E}^{2}}\right] \gamma^{4} Y(z)
$$

where $\mathcal{E}=\gamma m_{e} c^{2}$ is the particle energy, and $d n(\mathcal{E}) / d \mathcal{E}$ is the electron energy spectrum; $z=\left(\frac{3}{2} \nu / \nu_{\text {syn }}\right)^{2 / 3}$ is a normalized frequency, where $\nu_{\mathrm{syn}}=3 e B \gamma^{2} / 4 \pi m_{e} c$ is the characteristic synchrotron frequency for electrons with Lorentz factor $\gamma$. The function $Y(z)$ is defined as

$$
Y(z)=\frac{z^{2}}{2} \mathcal{P}\left\{\int_{-\infty}^{\infty} \frac{d \nu}{\nu} \exp \left(i \nu z+\frac{i \nu^{3}}{3}\right)\right\}+\frac{3 z^{2}}{2} \int_{-\infty}^{\infty} d \nu \nu \exp \left(i \nu z+\frac{i \nu^{3}}{3}\right)+z^{2} \int_{0}^{\infty} d \nu \nu \cos \left(\nu z+\frac{\nu^{3}}{3}\right)
$$

where $\mathcal{P}$ denotes Cauchy's principal value of the integral.

Since we are interested in $h$, only the real part of $Y(z)$ matters, and it can be easily shown that in fact only the last integral contributes. At frequencies $\nu \ll \nu_{m}, \kappa_{11}$ is dominated by electrons at the peak of the distribution function. We therefore take $d n(\mathcal{E}) / d \mathcal{E} \propto \delta\left(\mathcal{E}-\gamma_{m} m_{e} c^{2}\right)$. Expanding $\operatorname{Re}[Y(z)]$ at the limit $z \ll 1$, we finally obtain

$$
h_{\mathrm{rel}} \simeq \frac{e^{4} n_{e} B^{2} \sin ^{2} \vartheta}{4 \pi^{2} m_{e}^{3} c^{3} \nu^{3}} \frac{\gamma_{m}}{2}\left[\frac{\nu}{\nu_{m}\left(\gamma_{m}\right)}\right]^{4 / 3} \quad\left(\nu \ll \nu_{m}\right) .
$$


At frequencies $\nu>\nu_{m}$, the value of $h_{\mathrm{rel}}$ is sensitive to the shape of the electron distribution function. Specifically, for a powerlaw distribution, we simplify equation (20) of Sazonov (1969) to get the second of equations (6),

$$
h_{\mathrm{rel}} \simeq \frac{e^{4} n_{e} B^{2} \sin ^{2} \vartheta}{4 \pi^{2} m_{e}^{3} c^{3} \nu^{3}}\left(\frac{2}{p-2}\right) \gamma_{m}^{-(p-2)}\left(\frac{\nu}{\nu_{m}}\right)^{(p-2) / 2} \quad\left(\nu>\nu_{m}\right) .
$$

\section{A2. EMISSIVITY AND ABSORPTION COEFFICIENTS FOR SYNCHROTRON RADIATION}

For completeness, we provide expressions for the emissivity and absorption coefficient of the synchrotron radiation, using the notation $x=\nu / \nu_{\text {syn }}$ [see, e.g. Pacholczyk (1977), but note the typos in this source]:

$$
\begin{aligned}
\left(\begin{array}{c}
\varepsilon_{I} \\
\varepsilon_{Q} \\
\varepsilon_{V}
\end{array}\right) & =\frac{\sqrt{3}}{4 \pi} \frac{e^{3} B}{m_{e} c^{2}} \sin \vartheta \int_{0}^{\infty} \frac{d n\left(\mathcal{E}_{e}\right)}{d \mathcal{E}_{e}}\left(\begin{array}{c}
x \int_{x}^{\infty} K_{5 / 3}(z) d z \\
x K_{2 / 3}(x) \\
\left.\frac{2 \cot \vartheta}{\gamma_{e}}\left[\begin{array}{c}
x K_{1 / 3}(x)+\int_{x}^{\infty} K_{1 / 3}(z) d z
\end{array}\right)\right) d \mathcal{E}_{e}
\end{array}\right) \\
\left(\begin{array}{c}
\kappa \\
q
\end{array}\right) & =-\frac{\sqrt{3}}{8 \pi} \frac{e^{3} B}{m_{e}} \sin \vartheta \frac{1}{\nu^{2}} \int_{0}^{\infty} \mathcal{E}_{e}^{2} \frac{d}{d \mathcal{E}_{e}}\left(\frac{d n\left(\mathcal{E}_{e}\right) / d \mathcal{E}_{e}}{\mathcal{E}_{\mathcal{E}}^{2}}\right)\left(\begin{array}{c}
x \int_{x}^{\infty} K_{5 / 3}(z) d z \\
x K_{2 / 3}(x)
\end{array}\right) d \mathcal{E}_{e}
\end{aligned}
$$

Note that for a synchrotron source $\varepsilon_{U}=0, u=0$ (Pacholczyk 1977), and we may neglect $v$ in comparison to $\kappa$ and $q$.

Akerlof, C. W., et al. 1999, Nature, 398, 400

Berger, E., Kulkarni, S. R., \& Frail, D. A. 2003, ApJ, 590, 379

Blandford, R. D., \& McKee, C. F. 1976, Phys. Fluids, 19, 1130

Bloom, J. S. 2004, in ASP Conf. Ser. 312, Third Rome Workshop on Gamma-

Ray Bursts in the Afterglow Era, ed. M. Feroci, et al. (San Francisco: ASP), in press

Bloom, J. S., Frail, D. A., \& Kulkarni, S. R. 2003, ApJ, 594, 674

Bloom, J. S., Kulkarni, S. R., \& Djorgovski, S. G. 2002, AJ, 123, 1111

Chevalier, R. A., \& Li, Z.-Y. 1999, ApJ, 520, L29

Coburn, W., \& Boggs, S. E. 2003, Nature, 423, 415

Frail, D. A., Waxman, E., \& Kulkarni, S. R. 2000, ApJ, 537, 191

Frail, D. A., et al. 2001, ApJ, 562, L55

Freedman, D. L., \& Waxman, E. 2001, ApJ, 547, 922

Galama, T. J., et al. 1998, ApJ, 500, L97

Granot, J. 2003, ApJ, 596, L17

Gruzinov, A., \& Waxman, E. 1999, ApJ, 511, 852

Hjorth, J., et al. 2003, Nature, 423, 847

Lazzati, D., Ghisellini, G., Celotti, A., \& Rees, M. J. 2000, ApJ, 529, L17

Livio, M., \& Waxman, E. 2000, ApJ, 538, 187

Lyutikov, M., Pariev, V. I., \& Blandford, R. D. 2003, ApJ, 597, 998

Matsumiya, M., \& Ioka, K. 2003, ApJ, 595, L25

Medvedev, M. V., \& Loeb, A. 1999, ApJ, 526, 697

\section{REFERENCES}

Mészáros, P. 2002, ARA\&A, 40, 137

Mészáros, P., \& Rees, M. J. 1997, ApJ, 476, 232

Mészáros, P., Rees, M. J., \& Papathanassiou, H. 1994, ApJ, 432, 181

Nakar, E., Piran, T., \& Waxman, E. 2003, J. Cosmol. Astropart. Phys., 10, 005

Pacholczyk, A. G. 1977, Radio Galaxies (Oxford: Pergamon)

Piran, T. 2000, Phys. Rep., 333, 529

Rutledge, R. E., \& Fox, D. B. 2004, MNRAS, 350, 1288

Sagiv, A., \& Waxman, E. 2002, ApJ, 574, 861

Sazonov, V. N. 1969, Soviet Phys. JETP, 29(3), 578

Stanek, K. Z., et al. 2003, ApJ, 591, L17

Waxman, E. 1997a, ApJ, 485, L5 -. 1997b, ApJ, 491, L19

2003a, in Supernovae and Gamma Ray Bursters, ed. K. W. Weiler (Berlin: Springer), 393 2003b, Nature, 423, 388

Waxman, E., \& Draine, B. T. 2000, ApJ, 537, 796

Waxman, E., \& Loeb, A. 1999, ApJ, 515, 721

Wijers, R. A. M. J., \& Galama, T. J. 1999, ApJ, 523, 177

Willis, A. J. 1991, in Wolf-Rayet Stars and Interrelations with Other Massive Stars in Galaxies, ed. K. A. van der Hucht \& B. Hidayat (Dordrecht: Kluwer), 265

Zhang, B., Kobayashi, S., \& Mészáros, P. 2003, ApJ, 595, 950 